\title{
Deformación del Pico en el Caracara Común (Caracara cheriway), Tratamiento y Recuperación
}

\section{Francisco Aceituno'}

Resumen. Se reporta el registro de un caracara común (Caracara cheriway) con una deformación en el pico. El ave fue recibida en el Zoológico Rosy Walther en Tegucigalpa, Honduras. Esta anormalidad del pico no permitía que el ave se alimentara de forma normal ya que la maxila medía $12.5 \mathrm{~cm}$ y la mandíbula $5.5 \mathrm{~cm}$. No se determinó la causa específica de la deformación. Al ave la mantuvieron ilegalmente como mascota y generalmente en estas condiciones la alimentación ofrecida a las especies no suple sus requerimientos alimenticios y puede provocar este tipo de deformaciones. Para tratar esta deformación se utilizó una herramienta Dremel, lo que permitió corregir la forma y el tamaño del pico.

Palabras clave: Cautiverio, Dremel, Honduras, rapaces.

\section{Beak Deformation in Crested Caracara (Caracara cheriway), Treatment and Recovery}

Abstract. This is a report of a crested caracara with a bill deformation. The bird was received by Rosy Walther zoo in Tegucigalpa, Honduras. The abnormality did not allow the bird to feed normally considering that the maxillary beak measured $12.5 \mathrm{~cm}$ and the mandible beak $5.5 \mathrm{~cm}$. The specific cause of the abnormality was not identified. The bird was kept illegally as a pet and usually, in these feeding conditions, what is offered to the bird does not complete their dietary requirements and this can cause this kind of deformations. In order to treat the bill deformation a Dremel tool was used which allowed us to correct the bill size and shape.

Key words: Captivity, Dremel, Honduras, raptor.

\section{Introducción}

El pico de las aves está cubierto por una formación de tejido corneo que se denomina ranfoteca la cual está compuesta por la rinoteca, la parte superior o maxilar, y gnatoteca, la parte inferior o mandibular, ambas estructuras con un proceso de crecimiento constante (Vilar et al. 2010). Su contextura es similar a la de un hueso dérmico y su uso está relacionado con funciones como la alimentación, la manipulación de objetos, la comunicación social y el acicalamiento (Bennet 2011, Blancas-Calva 2013).

En las aves rapaces, la forma del pico determina el tipo de presas de las cuales se alimentan, por lo que la mayoría posee picos con ganchos fuertes y bordes filosos que les permiten rasgar carne (Samour 2006). De acuerdo a las diferentes estrategias de forrajeo de estas especies, el pico juega un papel determinante en su alimentación de modo que cualquier anormalidad puede afectar negativamente su sobrevivencia.
Las causas de las deformaciones en el pico de las aves están asociadas a factores genéticos, accidentes y enfermedades. Otra posible causa está relacionada con la mala nutrición de las especies en cautiverio (Pomeroy 1962, Craves 1994). Estas deformaciones generalmente resultan en un crecimiento anormal de la ranfoteca, gnatoteca 0 ambas, esto limita la capacidad para alimentarse de una forma adecuada (Craves 1994).

En vida silvestre, Craves (1994) documentó este fenómeno en aves paseriformes, Icteridae y Mimidae fueron las familias más frecuentes con este tipo de anomalías. En Alaska se registraron anormalidades en el pico de 30 especies, hubo altas proporciones en las especies Poecile atricapillus y Corvus brachyrhynchos (Collen et al. 2010). En aves rapaces se ha reportado este tipo de deformación en Buteo jamaicensis, Buteo lineatus, Falco peregrino y Falco sparverius; en su mayoría están asociadas a la exposición a sustancias químicas contaminantes (Strobel y Haralson-Strobel 2009, Collen et al. 2010,

\footnotetext{
${ }^{1}$ Sub Unidad de Biología y Veterinaria, Zoológico Rosy Walther, Secretaría de Energía, Recursos Naturales, Ambiente y Minas. Tegucigalpa, Honduras. E-mail: aceitunof@yahoo.com
} 
Tinajero y Rodríguez-Estrella 2011). En esta nota presento el primer registro de una deformación del pico de un caracara común (Caracara cheriway) en Honduras, además documento el tratamiento y recuperación del individuo.

\section{Descripción}

El espécimen (Figura 1) ingresó en julio de 2015 como donación, este había sido mantenido cautivo de forma ilegal en un almacén comercial. Una vez que se completó la ficha de recepción y mediante restricción física se evaluó clínicamente para determinar el estado de salud del ave. Se determinó que el individuo era un macho, no presentaba lesiones corporales visibles, pesaba $1.03 \mathrm{~kg}$ y su pico midió $12.5 \mathrm{~cm}$ la rinoteca y $5.5 \mathrm{~cm}$ la gnatoteca. El plumaje exhibía una coloración opaca, además se observó que el caracara no podía volar.

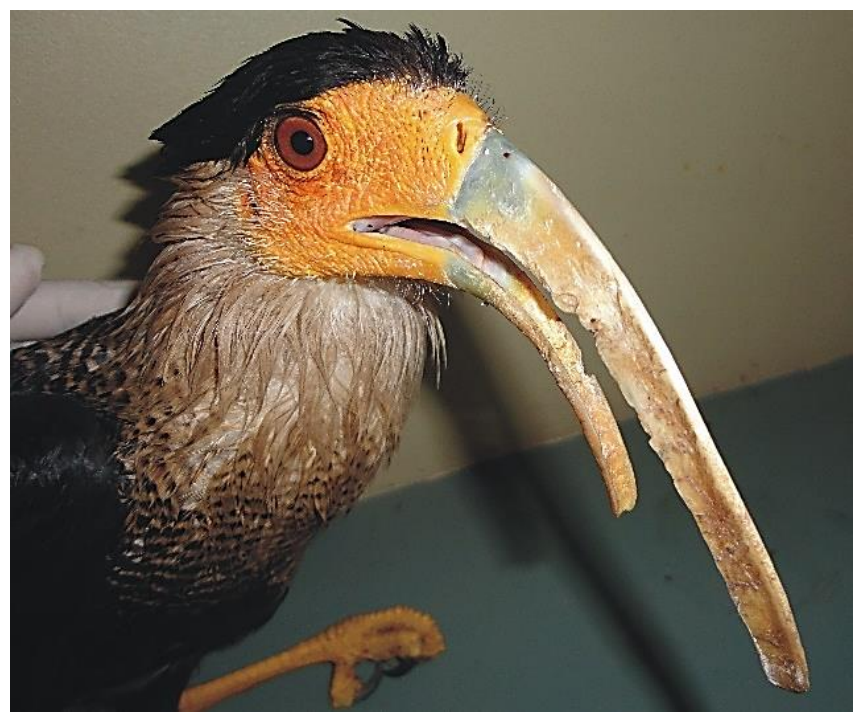

Figura 1. Individuo de caracara común (Caracara cheriway) con el pico deforme ingresado al Zoológico Rosy Walther en Tegucigalpa, Honduras.

Después de la revisión, el ave se alimentó a mano con ratones caseros (Mus musculus) y se remitió a un recinto de $1.60 \mathrm{~m}$ de ancho por $1.60 \mathrm{~m}$ de largo y $2.5 \mathrm{~m}$ de alto. Después de un día de estancia en el hospital médico veterinario se determinó corregir la deformación cortando y limando el pico. Para esta labor se utilizó una herramienta Dremel multiuso con un accesorio giratorio.

\section{Tratamiento y Recuperación}

El procedimiento fue en tres sesiones en un mes, la primera y segunda etapa consistió en el corte del pico, la cual se realizó por medio de contención manual sin necesidad de anestesiar al animal. Para la tercera etapa se limó y dio forma al pico (Figura 2), el ave fue sedada con Midazolam (0.1 mg/kg) y anestesiada con Ketamina $(0.1 \mathrm{mg} / \mathrm{kg})$.

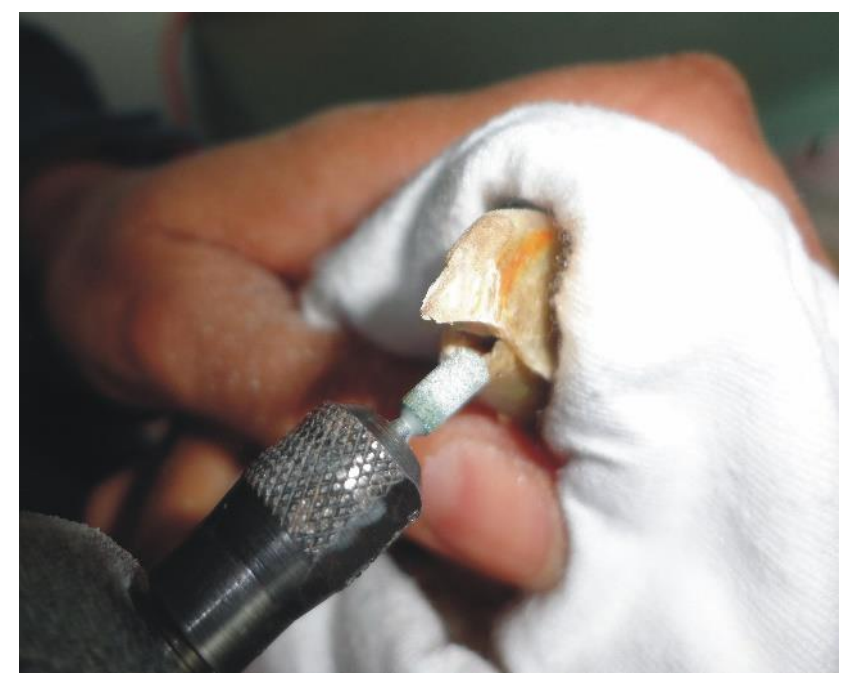

Figura 2. Corte y limado del pico deforme del caracara común (Caracara cheriway) ingresado al Zoológico Rosy Walther en Tegucigalpa, Honduras.

Cuando el animal ingresó al zoológico no era capaz de alimentarse por sí mismo, no desgarraba y solamente engullía el alimento. En consecuencia, y como parte del proceso, se realizaron observaciones ad libitum para determinar la recuperación del individuo. Después 30 días de la intervención se constató la mejora significativa al utilizar el pico para alimentarse (Figura 3). 


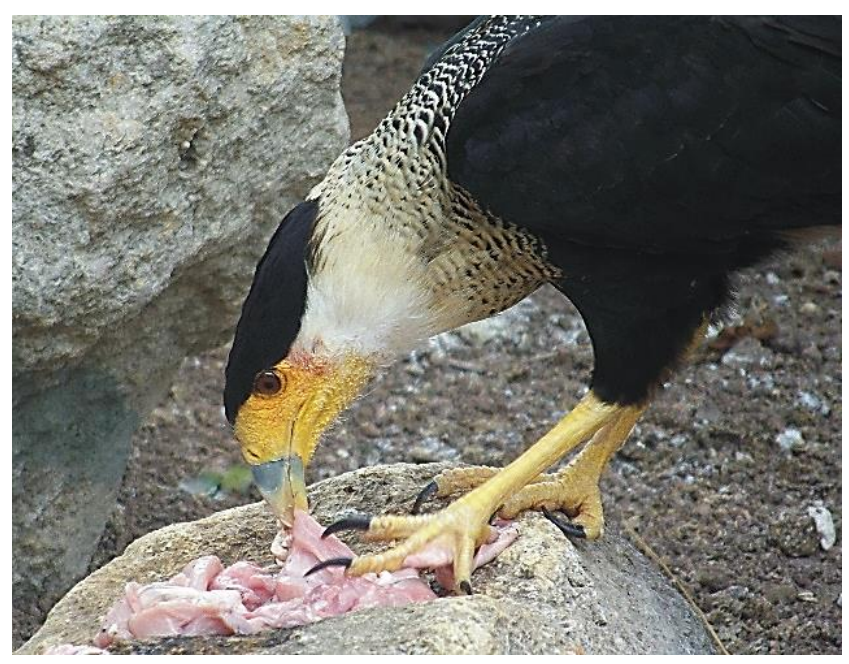

Figura 3. Individuo de caracara común (Caracara cheriway) alimentándose después del corte y limado del pico en el Zoológico Rosy Walther en Tegucigalpa, Honduras.

\section{Discusión}

El Caracara cheriway tiene una amplia distribución desde Estados Unidos hasta tierra del fuego. Es una especie generalista que aprovecha una diversidad de alimentos que incluyen carroña, pequeños vertebrados, reptiles, insectos, frutas y huevos (Rodríguez-Estrella y Rivera- Rodríguez 1997, Travaini et al. 2001). Aunque la especie posee un nicho alimentario amplio, una deformación de esta naturaleza sin duda afecta su capacidad de alimentarse y utilizar el pico para otras actividades.

Antes de su ingreso al zoológico, el ejemplar estuvo en cautiverio, la posible causa de esta anomalía puede estar relacionada con una alimentación deficiente que no cubría los requerimientos de la especie. Las aves rapaces en cautiverio pueden sufrir deficiencias nutricionales, especialmente de ciertas vitaminas y calcio, esto provoca que el pico tienda a doblarse limitando sus funciones normales (Keymer 1972).

Una vez que se observó que el animal recuperó las funciones normales del pico, se evaluó para establecer el destino final del mismo, determinando que no era viable su liberación al considerar que no se conocía con certeza la localidad de dónde provino y que por efecto del cautiverio se había habituado (impronta) a la presencia humana. En consecuencia, el individuo fue trasladado a un recinto de $6 \mathrm{~m}$ de ancho, $6 \mathrm{~m}$ de largo y $2 \mathrm{~m}$ de alto, como parte de la colección de animales de exhibición del zoológico. La reinserción de un animal extraído del medio silvestre es un proceso complejo por lo que las opciones para individuos decomisados son el cautiverio, la liberación o la eutanasia.

Para tomar la opción de la liberación y que esta sea exitosa, se debe considerar aspectos como el grado de amenaza de la especie, su contribución a la conservación de la misma, posibles enfermedades y el origen de los animales incautados (UICN 2000). Por tal razón, es importante fortalecer la implementación de la normativa legal vigente para controlar esta problemática, así mismo es necesario el diseño e implementación de campañas de educación ambiental orientadas a prevenir la compra y tenencia ilegal de fauna silvestre como mascotas.

Este registro permite ampliar el conocimiento de la incidencia de este tipo de deformaciones en el pico de aves rapaces en Honduras. En este sentido, es importante documentar estas anomalías para determinar sus posibles causas y las repercusiones para estas especies.

\section{Literatura Citada}

Bennett, R.A. 2011. Surgery of the avian beak. In: Annual Conference \& Expo With the Association of Mammal Veterinarians: Proceedings of the Association of Avian Veterinarians. Seattle. p. 191-195.

Blancas-Calva, E. 2013. Un caso de deformación del pico en el tordo cabeza café (Molothrus ater). Huitzil 14 (1):75-78.

Craves, J.A. 1994. Passerines with deformed bills. North American Bird Bander 19:14-18.

Collen, M.H., L.M. Pajot, S.M. Matsuoka, C.V. Hemert, J. Terenzi, S.L. Talbot, D.M. Mulcahy, C.U. Meteyer y K.A. Trust. 2010. Epizootic of beak deformities among wild birds in Alaska: an emerging disease in North America. The Auk 127:882-898.

Keymer, I.F. 1972. Diseases of birds of prey. Veterinary Record 90:579-594.

Pomeroy, D.E. 1962. Birds with abnormal bills. British Birds 56:49-72. 
Rodríguez-Estrella, R., y L.B. Rivera-Rodríguez. 1997. Crested caracara food habits in the Cape Region of Baja California, Mexico. Journal of Raptor Research 31:228-233.

Samour, J. 2006. Management of raptors. In: Clinical Avian Medicine. G. Harrison, T. Lightfoot (eds.). Volumen 2. Spix Publishing. p. 915-956.

Strobel, B.N. y C.L. Haralson-Strobel. 2009. Foraging by a red-shouldered hawk (Buteo lineatus) with severe bill damage. Bulletin of Texas Ornithological Society 42:9698.

Tinajero, R. y R. Rodríguez-Estrella. 2011. Halcón cola roja con pico anormal, un registro notable para Baja California Sur, México. Acta Zoológica Mexicana 27(3):825-828.
Travaini A., J.A. Donazar, O. Ceballos y F. Hiraldo. 2001. Food habits of the Crested caracara (Caracara plancus) in the Andean Patagonia: the role of breeding constraints. Journal of Arid Environments 48:211-219.

UICN. 2000. Guías de la UICN para la disposición de animales confiscados. Aprobadas en la $51^{\mathrm{a}}$ Reunión del consejo de la UICN, Gland, Suiza.

Vilar, J.M, G. Altilia y G. Spinella. 2010. Aspectos clínicos en la reparación del pico en un Cálao (Aceros plicatus, Foster 1781) mediante dos técnica diferentes. Revista Electrónica de Veterinaria 11(1):1-5.

Recibido para publicación 22 de octubre del 2015. Aceptado para publicación el 8 de enero del 2016. 